\title{
Sprouty 4 mRNA variants and protein expressions in breast and lung-derived cells
}

\author{
ANGELINA DORIGUZZI, JIHEN SALHI and HEDWIG SUTTERLÜTY-FALL \\ Institute of Cancer Research, Comprehensive Cancer Center, Department of Medicine I, \\ Medical University of Vienna, A-1090 Vienna, Austria
}

Received March 29, 2016; Accepted July 22, 2016

DOI: $10.3892 / \mathrm{ol} .2016 .5216$

\begin{abstract}
Sprouty proteins are modulators of mitogen-induced signalling processes and are therefore hypothesized to affect malignant diseases. As a member of the Sprouty family, Sprouty4 has been previously shown to function as a tumour suppressor in lung and breast cancer. The present study analysed the expression of two known Sprouty4 splice variants in cells established from malignant and normal lung and breast tissues using semi-quantitative reverse transcription-polymerase chain reaction and immunoblotting. The results indicated that the expression of the two messenger RNA (mRNA) variants was reduced in the cells derived from malignant tissue in comparison to the normal counterparts. Although the expression of the two splice variants were associated in both tissue types, on average, the relative expression of the longer variant was slightly increased in malignant cells compared with normal tissues. Notably, the protein levels reflected the expression observed at the mRNA level only in breast-derived cells. Contrarily, with regards to the measured mRNA levels, Sprouty4 protein was disproportional augmented in lung cells known to harbour the mutated K-Ras gene.
\end{abstract}

\section{Introduction}

The alteration of important molecules involved in signal transduction is a common characteristic of all malignant cells (1). The mitogen activated protein kinase (MAPK) pathway, which is crucially involved in balancing cell

Correspondence to: Dr Hedwig Sutterlüty-Fall, Institute of Cancer Research, Comprehensive Cancer Center, Department of Medicine I, Medical University of Vienna, 8a Borschkegasse, A-1090 Vienna, Austria

E-mail: hedwig.sutterluety-fall@meduniwien.ac.at

Abbreviations: RT-PCR, reverse transcription-polymerase chain reaction; Spry, sprouty; nHMECs, normal human mammary epithelial cells; nHBEpCs, normal human bronchial epithelial cells

Key words: sprouty, breast cancer, lung cancer, splice variants, K-Ras death and differentiation on one side and proliferation and migration on the other, is one of the best described signalling routes that is deregulated in cancer (2). Due to the vital role of the MAPK pathway, numerous feedback loops are present to allow a fine-tuning of signal intensity and duration (3). Certain molecules acting in such a regulatory loop are the members of the Sprouty (Spry) protein family. First discovered in Drosophila $(4,5)$, the Spry proteins counteract processes induced by the presence of various growth factors. In humans, 4 Spry family members are known; all of which share a conserved Spry domain at the C-terminus and 2 homolog N-terminal boxes (6). As indicated by knockout studies in mice, the functions of Sprouty proteins overlap but are not redundant (7-10). Although Spry1, Spry2 and Spry4 can be detected in all organs, their expression pattern in the various cell types is clearly distinguishable (11). Like in Drosophila, mammalian Spry proteins interfere with signal transduction, particularly in the MAPK pathway, in response to various mitogens, such as the members of the fibroblast growth factors (6). In contrast to Drosophila, in mammalian systems, signalling activated by epidermal growth factor is increased with augmented Spry expression. Therefore, the Spry proteins in humans are generally considered as modulators of signal transduction (12).

Accordingly, dependent on the tumour type, Spry proteins are found to fulfil multiple roles in cancer. For example, Spry2 has been indicated to function as an oncogene in colon cancer $(13,14)$ and Spryl has a tumour promoting role in rhabdomyosarcoma (15). However, Spry proteins have been primarily shown to suppress the tumorigenic process (12). Spry2 is a tumour-suppressor in lung $(16)$, prostate $(17,18)$, breast (19), liver (20) and ovarian cancer (21). In addition, Spryl has also been identified as negative regulator of cancerogenesis in numerous types of tumour, including breast (19), prostate (22) and ovarian cancer (23). Although Spry4 has been less intensively studied in comparison to Spry2 and Spry1, the literature documents that the likelihood of Spry4 to act as a tumour suppressor may be dependent on the cancer origin. Spry4 has previously failed to significantly influence malignant phenotypes in cancer originating from the bones (24), ovary (21) or pancreas (25), while it fulfils all features of a tumour suppressor when the cancer has developed from lung (26) or breast tissue (27) cells. This includes an overall decrease in Spry4 expression in cancerous tissues 
compared with the normal tissue counterparts $(26,28)$. However, the mechanisms responsible for this decreased expression are required to be elucidated.

The gene encoding Spry4 is localized on chromosome 5q and is organized in 3 exons (29). Due to alternative splicing, two messenger RNA (mRNA) variants can be generated. Spry4.1 is missing the second exon, while the longer variant Spry4. 2 consists of all 3 exons, and the two variants are shown to be expressed in most organs (30). Since alternative splicing is often involved in modulating gene expression and function during the cancerogenic process, the present study aimed to analyse the expression pattern of the two Spry4 variants in breast and lung cancer-derived cells.

\section{Materials and methods}

Cell culture. All lung adenocarcinoma-derived (Calu3, Calu6, A549, A427 and SKLU-1) and breast cancer-derived (MDA-MB231, BT20, MDA-MB468, ZR-75, MCF7 and SKBR3) cell lines were purchased from American Type Culture Collection (ATCC; Rockville, MD, USA). The squamous cell carcinoma-derived cell lines (VL-7, VL-8 and VL-10) were established at the Institute of Cancer Research, Comprehensive Cancer Center in the Department of Medicine I, Medical University of Vienna (Vienna, Austria) (31). Of the lung cancer-derived cell lines, 3 (A549, Calu6 and A427) were known to harbour K-Ras mutations (16). Additionally, the embryonic lung fibroblasts (WI-38) and the fibrosarcoma-derived HT-1080 cell line were obtained from ATCC. All the cells were maintained in Dulbecco's modified Eagle's medium (Gibco; Thermo Fisher Scientific, Inc., Waltham, MA, USA) supplemented with $10 \%$ fetal calf serum (FCS; Thermo Fisher Scientific, Inc.), penicillin (100 units $/ \mathrm{ml})$ and streptomycin $(100 \mu \mathrm{g} / \mathrm{ml})$ at $37^{\circ} \mathrm{C}$ in $7.5 \%$ $\mathrm{CO}_{2}$. Primary normal human mammary epithelial cells (nHMECs) were purchased from Thermo Fisher Scientific, Inc. and normal human bronchial epithelial cells (nHBEpCs) from Pelobiotech GmbH (Planegg, Germany). These cells were maintained according to the providers' recommendations.

Reverse transcription-polymerase chain reaction (RT-PCR). RNA was extracted as previously described (32). Total RNA $(3 \mu \mathrm{g})$, random hexamers $(100 \mathrm{ng})$ and moloney-murine leukemia virus reverse transcriptase were used to generate of complementary DNA, according to manufacturer's protocol (Promega, Madison, USA). Semi-quantitative PCR was performed as previously described (31). For Spry4.1, the primer 5'-CCCGGCTTCAGGATTTAC-3' (forward) was used, and for Spry4.2, the primer 5'-AGCCTGTATTGAGCG GTTTG-3' (forward) was used. The primer 5'-GCTGGACCA TGACTGAGTTG-3' (reverse) was used for the two variants. As a control for standardisation, $\beta$-microglobulin levels were measured using the following primer pair: 5'-ACCCCAACT GAAAAAGATGA-3' (forward) and 5'-ATCTTCAAACCT CCATGATG-3' (reverse). The thermocycling conditions were as follows: Initial denaturation step at $95^{\circ} \mathrm{C}$ for $2 \mathrm{~min}$, followed by different numbers of cycles ( 30 and 33 cycles for Spry4.1, 35 and 38 cycles for Spry 4.2 and 30 cycles for $\beta$-microglobulin) consisting of $95^{\circ} \mathrm{C}$ for $30 \mathrm{sec}$, annealing at $58^{\circ} \mathrm{C}$ for $30 \mathrm{sec}$ and extension at $72^{\circ} \mathrm{C}$ for $1 \mathrm{~min}$. The PCR reaction was terminated with a final step at $72^{\circ} \mathrm{C}$ for $5 \mathrm{~min}$. For analysis, the PCR products were separated by polyacrylamide gel electrophoresis, stained with ethidium bromide and scanned using the Gel Doc ${ }^{\mathrm{TM}}$ XR Imaging System (Bio-Rad Laboratories, Hercules, CA, USA). Quantification of the obtained signals was performed using Image Quant 5.0 software (GE Healthcare Life Sciences, Chalfont, UK). $\beta$-microglobulin was used to standardise the RNA input. For normalisation, RNA of an arbitrarily chosen, external cell line (HT-1080) was included in each experiment and set as 1 (31).

Immunoblot. Protein levels were determined and extracted as previously described (33). Immunoblotting was carried out using affinity-purified antibodies against Spry4 (1:100) that were generated as previously described (30). Antibodies against glyceraldehyde 3-phosphate dehydrogenase (GAPDH; clone, G-9; mouse monoclonal; catalog no., sc-365062; 1:30,000; Santa Cruz Biotechnology Inc., Dallas, TX, USA) and horseradish peroxidase-coupled sheep anti-mouse (catalog no., NA931; 1:5,000) and donkey anti-rabbit (catalog no., NA934; 1:5,000) IgG secondary antibodies (GE Healthcare Life Sciences) were also used.

Statistical analysis. All data were presented as the mean value \pm standard error of the mean. GraphPad Prism 5 software (GraphPad Software Inc., La Jolla, CA, USA) was used to perform all statistical analyses. Expression differences were calculated using an unpaired Student's $t$-test. Associations were analysed assuming a Gaussian population. $\mathrm{P}<0.05$ was considered to indicate a statistically significant difference.

\section{Results}

Expression of Spry4.1 and Spry4.2 in breast-derived cells. In order to analyse the expression pattern of the two reported Spry4 splice variants, RNA from 6 breast cancer-derived cell lines and nHMECs was isolated. Using RT-PCR, the levels of the two Spry mRNA variants were determined. As depicted in Fig. 1, both Spry4 variants are expressed in all investigated cell lines. Compared with the levels detected in the non-malignant nHMEC cells, Spry4.1 expression in MDA-MB231 was elevated by approximately 2-3 fold. In all other breast tumour cell lines, the levels of the Spry4.1 variant were reduced by a third (BT20, MDA-MB468, ZR-75) or even a fifth (MCF7, SKBR3) (Fig. 1A). The longer Spry4 variant (Spry4.2), which includes exon 2, demonstrated similar results to the Spry4.1 variant, as the expression levels were decreased in all tumour-derived cell lines with the exception of MDA-MB231, although the differences were less pronounced (Fig. 1B). Consequently, the statistical analysis revealed that expression patterns of Spry4.1 and Spry4.2 are associated.

These data indicate that the mechanisms involved in repression of Spry 4 mRNA during malignant transformation of breast cells may not discriminate between the two Spry4 variants.

Expression of Spry4.1 and Spry4.2 in lung-derived cells. In addition to its involvement in breast cancer (27), Spry4 has also been shown to function as a tumour suppressor in lung 
A

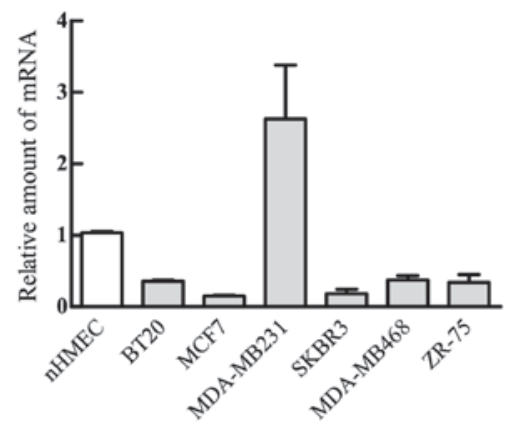

B

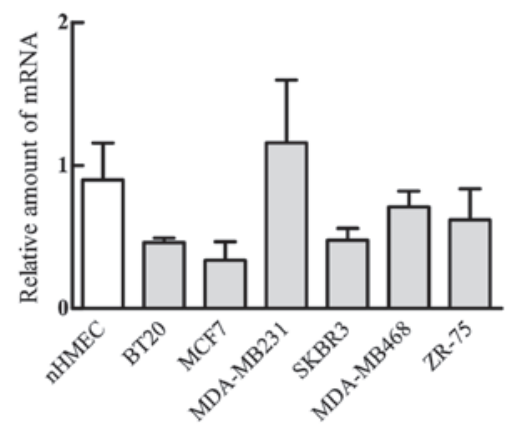

C

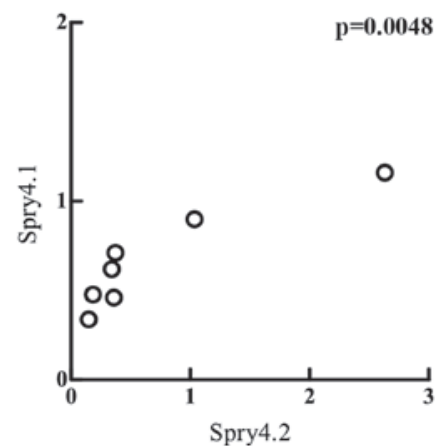

Figure 1. Expression of Spr4.1 and Spr4.2 variants in breast-derived cells. Total RNA of logarithmical growing breast-derived cells was isolated. Using semi-quantitative reverse transcription-polymerase chain reaction, mRNA levels of (A) Spry4.1 and (B) Spr4.2 in nHMECs (white bars) and breast cancer-derived cell lines (grey bars) were determined. Each bar summarizes the results obtained from three independent experiments. Mean values \pm standard error of the mean are shown. (C) Association using the mean values of Spry4.1 and Spry 4.2 mRNA variants was calculated using GraphPad Prism 5 software. The calculated P-value is indicated. mRNA, messenger RNA; Spry, sprouty; nHMEC, normal human mammary epithelial cell.

cancer (26). Therefore, the present study subsequently analysed the expression of each Spry4 variant in lung cancer-derived cell lines and compared the determined levels with those evaluated for nHBEpCs. The nHBEpCs were observed to express significantly more of the two Spry4 variants (Fig. 2A and B). Analysis of the Spry4.1 expression revealed that all tumour-derived cell lines irrespective of their histological subtype and the occurrence of K-Ras mutations show reduced Spry4 levels (Fig. 2A). Additionally, Spry4.1 levels determined for the lung adenocarcinoma-derived cell lines (Calu3, Calu6, A549, A427 and SKLU-1) were significantly increased compared with the levels measured in squamous cell carcinoma cell lines (VL-8, VL-7 and VL-10). Analogue to the Spry4.1 variant, Spry4.2 mRNA levels in nHBEpCs were increased compared with the levels measured in the embryonic lung fibroblasts (WI-38) and in the majority of the tumour-derived cell lines. Only A427 and SKLU-1 had levels comparable to the normal counterpart
A

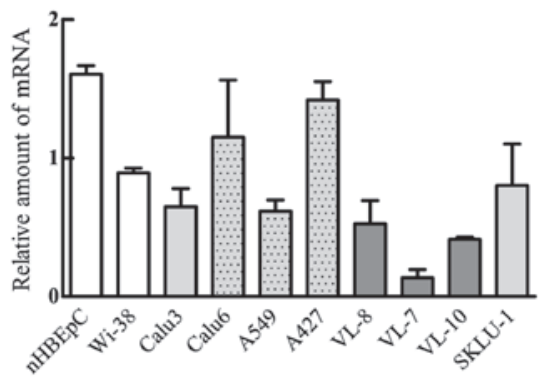

B

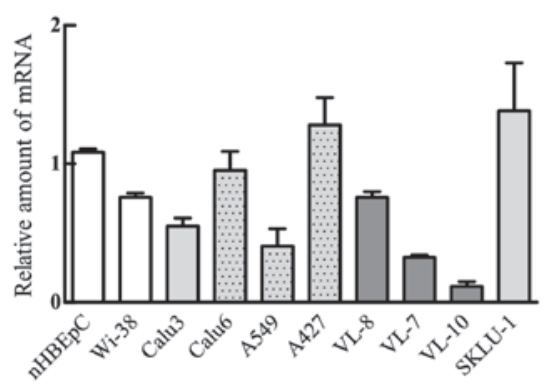

C

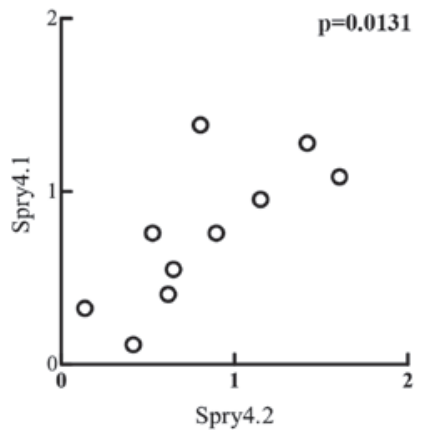

Figure 2. Expression of mRNA variants Spry4.1 and Spry4.2 in lung-derived cells. Total RNA isolated from logarithmical growing cells was used to determine the levels of (A) Spry4.1 and (B) Spr4.2 variants by semi-quantitative reverse transcription-polymerase chain reaction. Intensity of the signals was measured with Image Quant 5.0 software using $\beta$-microglobulin as an internal standard. HT-1080 served as an external standard for quantification. Mean values \pm standard error of the mean (from three independent experiments) of nHBEpCs (white bars) and lung cancer-derived cells (grey bars) are depicted. Cell lines derived from squamous cell carcinoma (dark grey) and adenocarcinoma (light grey) were included. Cell lines harbouring mutated Ras are highlighted using a dotted pattern. (C) GraphPad Prism 5 software was used to calculate the association between the mean values of Spry4.1 and Spry4.2 variant levels. The P-value is depicted at the right top of the graph. mRNA, messenger RNA; Spry, sprouty; nHBEpC, normal human bronchial epithelial cell.

(Fig. 2B). Hence the expression levels of the Spry4 splicing variants are also significantly associated in lung-derived cells (Fig. 2C). Therefore, the two Spry4 variants are hypothesized to be co-regulated.

Comparison of Spry4 mRNAs with protein levels in breast-derived cells. The second step of gene expression is the conversion of the mRNA to the protein. Due to numerous regulative processes, the patterns measured at the RNA level are not always reflected at the protein stage. In the case of Spry4 expression in breast-derived cells, the protein levels determined by immunoblotting were similar to those determined in RT-PCR. In nHMECs, Spry4 was found to be more abundant compared with all other breast cancer-derived cell 
A

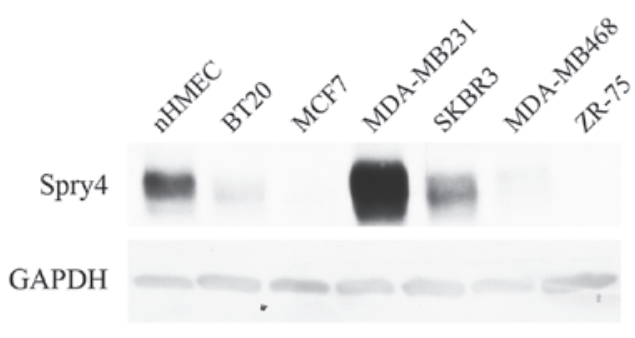

C

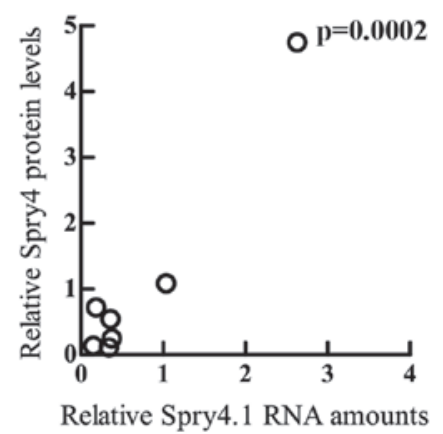

B

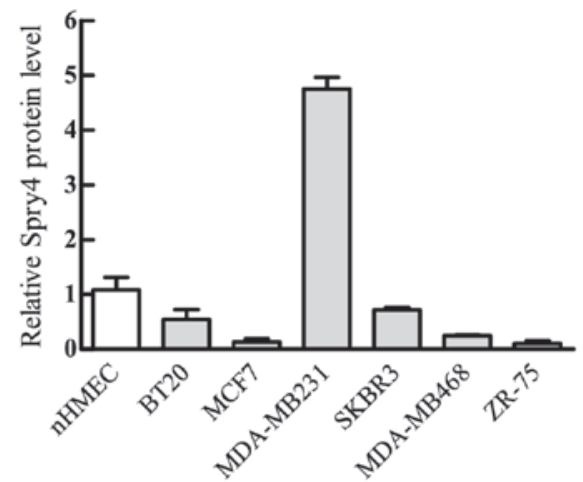

D

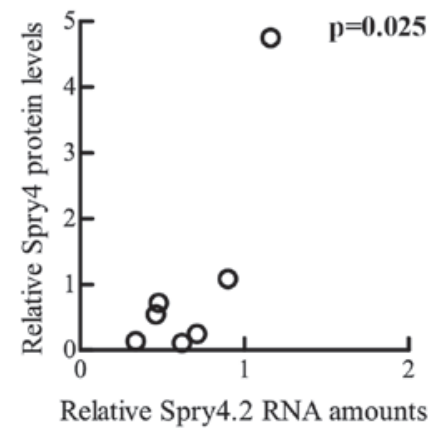

Figure 3. Comparison of Spry4 protein levels with the corresponding Spry4 mRNA in cells established from breast tissue. (A) Protein was isolated from logarithmical growing breast-derived cells and analysed by immunoblotting with the indicated antibodies. (B) Quantification of signals derived from three independent experiments was carried out using Image Quant 5.0 software. Spry4 protein levels were normalized to GAPDH expression and the additional determined value for the control cells (HT-1080) was set as 1 . Mean values \pm standard error of the mean of nHMECs (white bar) and the breast cancer-derived cell lines (grey bars) were depicted using GraphPad Prism 5 software. Spry4 protein expression in the investigated cell lines was compared to either (C) Spry4.1 or (D) Spry4.2 mRNA levels. The association was calculated using GraphPad Prism 5 software and the P-values were included in the graphs. Spry, sprouty; mRNA, messenger RNA; nHMEC, normal human mammary epithelial cell; GAPDH, glyceraldehyde 3-phosphate dehydrogenase.

A

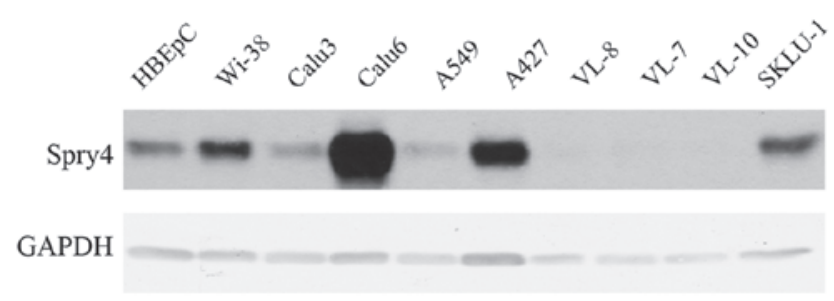

C

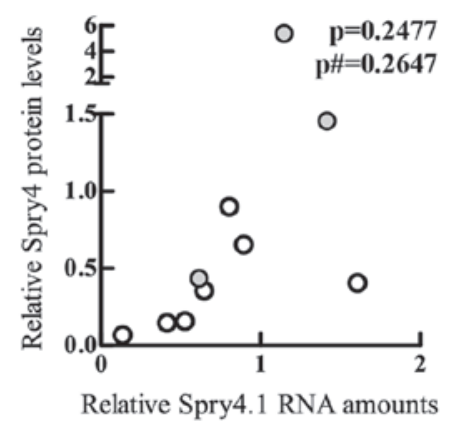

B

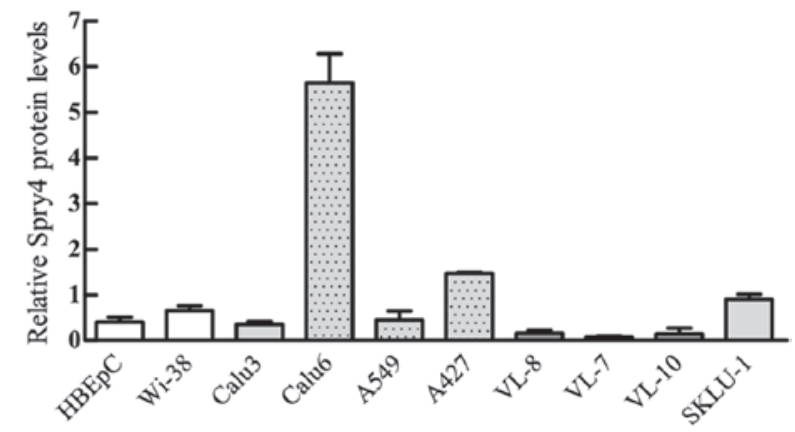

D

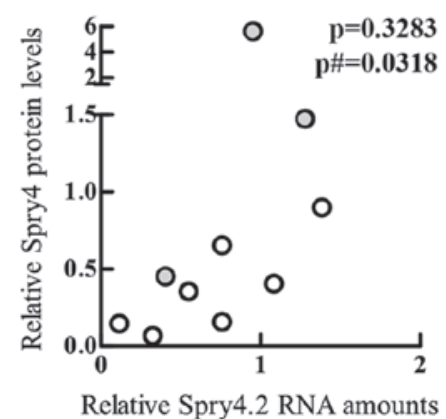

Figure 4. Levels of Spry4 protein in lung cells were compared with the respective Spry4.1 and Spry4.2 mRNA variants. (A) A representative immunoblot measuring Spry4 protein expression in various lung and lung cancer-derived cells in the logarithmic stage of growth is depicted. GAPDH was used as loading control. (B) Using Image Quant 5.0 software, Spry4 and GAPDH protein signals were quantified. The relative Spry4 protein levels were calculated using the GAPDH signals as a standardised protein loading and HT-1080 as an external standard set as 1. Mean values \pm standard error of the mean are depicted. nHBEpCs (white bars) were compared with cell lines established from adenocarcinoma with wild-type K-Ras (light grey), mutated K-Ras (light grey with a dotted pattern) and from squamous cell carcinoma (dark grey). (C) Mean protein levels were compared with Spry4.1 mRNA levels or (D) Spry4.2 variant expression. The cell lines harbouring mutated K-Ras are indicated in grey. Using GraphPad Prism 5 software, the association was calculated. In addition to the P-value summarizing all lung cells, the P-value excluding the K-Ras mutated cell lines is indicated. Spry, sprouty; mRNA, messenger RNA; nHBEpC, normal human bronchial epithelial cell; GAPDH, glyceraldehyde 3-phosphate dehydrogenase; p, P-value for all lung cells; p\#, P-value excluding K-Ras mutated cell lines. 
lines, with the exception of MDA-MB231 (Fig. 3A and B). Although the protein levels were associated with the expression of the two Spry4 variants, the differences were more similar to the amounts of Spry4.1 measured (Fig. 3C and D). This comparison suggests that the mechanisms regulating Spry4 gene expression subsequent to mRNA production are not involved in the malignant transformation process of breast cells.

Comparison of Spry 4 mRNAs with protein levels in lung-derived cells. Determination of Spry4 protein levels in lung-derived cells revealed that only the squamous cell carcinoma-derived cell lines have clearly decreased Spry4 protein levels compared with nHBEpCs (Fig. 4A and B). Two of the cell lines (Calu6 and A427) expressed definitively higher levels of Spry4 compared with nHBEpCs (Fig. 4A). The protein expression levels of the other adenocarcinoma cells were comparable to those measured in nHBEpC cells (Fig. 4A and B). This is in clear contrast to the observations made at mRNA level (Fig. 2). Accordingly, the expression pattern of Spry4 protein, Spry4.1 (Fig. 4C) and Spry4.2 (Fig. 4D) are not associated. Notably, the two cell lines (Calu6 and A427) showing clear elevated Spry4 protein expression are known to harbour an activating K-Ras mutation. Although the Spry4 protein levels in the third cell line with a mutated K-Ras, A549, are only comparable to the levels measured in normal cells, the protein expression is relatively high considering that the mRNA levels are decreased by half compared with that of the normal cells (compare Fig. 2A and B with Fig. 4B). If only the cells with an unaltered K-Ras are analysed, the protein levels are least significantly associated with the Spry4.2 variant. These results indicate that a constitutive signal transduction via K-Ras may affect the mechanisms involved in modulating Spry4 protein levels.

\section{Discussion}

During cancer development, signalling transduction cascades are often targeted by various alterations. Spry proteins modulate the intensity and duration of the transmitted signals within such cascades, and therefore are important for the tumorigenesis of various tissues. Spry4 has been previously shown to fulfil a tumour suppressive function in lung (26) and breast (27) cancer.

The present study investigated the expression levels of the two known Spry4 mRNA splice variants in cells that were cultured from the normal and malignant tissues of the lungs and breast. All of the cell lines used in the study expressed both forms. In accordance with a previous study (30), which investigated the Spry4 mRNA forms in various tissues, the shorter Spry4.1 mRNA was the more abundant variant in the present study. With regards to normal cells, expression of the two Spry4 forms was higher in lung-derived cells compared with the breast-derived cells. Although in a previous study the breast tissue was not included, Spry4 expression in the lung tissue was indicated to be increased compared with the majority of other organ tissues (30).

Furthermore, the present study compared the expression of Spry4 variants in nHMECs with the expression in breast cancer-derived cell lines and found that, with one exception, the expression of both variants was decreased in the malignant cells. In agreement with an earlier study (27), the Spry4 levels were found to be differentially expressed depending on the subtype. The luminal subtypes of breast cancer demonstrated a decreased expression of the two Spry4 variants compared with the basal subtype. Additionally, the present observations revealed that Spry4 mRNA levels are repressed in malignant lung cells in comparison to their normal counterparts, which reinforces the data from Tennis et al (26). Supplementary to the observations regarding breast and lung malignancies, repressed Spry4 mRNA levels have already been found in the liver (34) and in approximately half of the prostate cancers investigated (35).

In a previous study that investigated the Spry4 mRNA variants, qRT-PCR of 20 types of normal tissues showed that the levels of the two Spry4 mRNA variants were associated (29). Accordingly, in the lung and breast cells used in the present study, a statistically significant association between the short Spry4.1 and the slightly longer Spry4.2 version could be observed. These data indicate that the repression of Spry4 levels in malignant cells is not mainly achieved by alterations to the components that regulate splicing.

The present study also compared the mRNA and protein levels in the investigated cell lines. In breast-derived cells, mRNA and protein expression were associated. Although, to the best of our knowledge, the present study is the first to report that Spry4 mRNA and protein levels are associated across various cell lines that originate from one tissue, a concomitant increase of Spry4 mRNA production and protein expression in response to serum induction of malignant (36) and normal cells (37) has already been reported. Additionally, elevated Spry4 expression following the application of amphiregulin (38) and hypoxia (32) has been observed at the mRNA and protein levels.

The immunoblot analysis confirms that in breast tissues, the Spry4 protein is repressed in the cancer cells compared with their normal cell counterparts. In addition, previous results generated by immunohistochemistry have shown that, in the endometrium, Spry4 is decreased in the malignant tissue compared with normal tissues (39).

In contrast to the significant association of RNA and protein expression in breast cells, the mRNA levels in lung-derived cells do not determine the amount of Spry4 protein, indicating that posttranscriptional mechanisms have an important affect on Spry4 expression in lung tissue. Earlier observations support that Spry4 expression is also controlled at a protein level, since the amplitude of Spry4 protein increase in response to serum was more intense compared with at mRNA level, and epidermal growth factor-mediated signalling promoted Spry4 protein expression without affecting its mRNA levels (36). Particularly in cell lines harbouring a mutated version of the K-Ras gene, the Spry4 protein levels were notably higher than expected from the detected mRNA levels. This potentially suggests that one of the components involved in the regulation of the Spry4 at the protein level is controlled by K-Ras-connected pathways.

In conclusion, the presented study shows that, in breast and lung tissues, the expression of Spry4 splice variants is co-regulated, and is repressed in malignant cells. At protein level, the expression of Spry4 may be additionally upregulated by a K-Ras dependent mechanism.

\section{Acknowledgements}

The present study was supported by the Herzfelder'sche Family Foundation, Vienna, Austria (grant no. 2015). 


\section{References}

1. Hahn WC and Weinberg RA: Rules for making human tumor cells. N Engl J Med 347: 1593-1603, 2002.

2. Hommes DW, Peppelenbosch MP and van Deventer SJ: Mitogen activated protein (MAP) kinase signal transduction pathways and novel anti-inflammatory targets. Gut 52: 144-151, 2003.

3. Freeman M: Feedback control of intercellular signalling in development. Nature 408: 313-319, 2000.

4. Casci T, Vinós J and Freeman M: Sprouty, an intracellular inhibitor of Ras signaling. Cell 96: 655-665, 1999.

5. Hacohen N, Kramer S, Sutherland D, Hiromi Y and Krasnow MA sprouty encodes a novel antagonist of FGF signaling that patterns apical branching of the Drosophila airways. Cell 92: 253-263, 1998.

6. Guy GR, Jackson RA, Yusoff P and Chow SY: Sprouty proteins: Modified modulators, matchmakers or missing links? J Endocrinol 203: 191-202, 2009.

7. Basson MA, Akbulut S, Watson-Johnson J, Simon R, Carroll TJ, Shakya R, Gross I, Martin GR, Lufkin T, McMahon AP, et al: Sproutyl is a critical regulator of GDNF/RET-mediated kidney induction. Dev Cell 8: 229-239, 2005.

8. Shim K, Minowada G, Coling DE and Martin GR: Sprouty2, a mouse deafness gene, regulates cell fate decisions in the auditory sensory epithelium by antagonizing FGF signaling. Dev Cell 8 : 553-564, 2005.

9. Taketomi T, Yoshiga D, Taniguchi K, Kobayashi T, Nonami A, Kato R, Sasaki M, Sasaki A, Ishibashi H, Moriyama M, et al Loss of mammalian Sprouty2 leads to enteric neuronal hyperplasia and esophageal achalasia. Nat Neurosci 8: 855-857, 2005.

10. Taniguchi K, Ayada T, Ichiyama K, Kohno R, Yonemitsu Y Minami Y, Kikuchi A, Maehara Y and Yoshimura A: Sprouty2 and Sprouty4 are essential for embryonic morphogenesis and regulation of FGF signaling. Biochem Biophys Res Commun 352: 896-902, 2007.

11. Zhang S, Lin Y, Itäranta P, Yagi A and Vainio S: Expression of Sprouty genes 1, 2 and 4 during mouse organogenesis. Mech Dev 109: 367-370, 2001

12. Masoumi-Moghaddam S, Amini A and Morris DL: The developing story of Sprouty and cancer. Cancer Metastasis Rev 33: 695-720, 2014.

13. Barbáchano A, Ordóñez-Morán P, Garcia JM, Sánchez A, Pereira F, Larriba MJ, Martínez N, Hernández J, Landolfi S, Bonilla F, et al: SPROUTY-2 and E-cadherin regulate reciprocally and dictate colon cancer cell tumourigenicity. Oncogene 29: 4800-4813, 2010.

14. Holgren C, Dougherty U, Edwin F, Cerasi D, Taylor I, Fichera A, Joseph L, Bissonnette M and Khare S: Sprouty-2 controls c-Met expression and metastatic potential of colon cancer cells: Sprouty/c-Met upregulation in human colonic adenocarcinomas. Oncogene 29: 5241-5253, 2010.

15. Schaaf G, Hamdi M, Zwijnenburg D, Lakeman A, Geerts D, Versteeg R and Kool M: Silencing of SPRY1 triggers complete regression of rhabdomyosarcoma tumors carrying a mutated RAS gene. Cancer Res 70: 762-771, 2010

16. Sutterluty H, Mayer CE, Setinek U, Attems J, Ovtcharov S, Mikula M, Mikulits W, Micksche $M$ and Berger W: Down-regulation of Sprouty2 in non-small cell lung cancer contributes to tumor malignancy via extracellular signal-regulated kinase pathway-dependent and -independent mechanisms. Mol Cancer Res 5: 509-520, 2007.

17. McKie AB, Douglas DA, Olijslagers S, Graham J, Omar MM, Heer R, Gnanapragasam VJ, Robson CN and Leung HY: Epigenetic inactivation of the human sprouty2 (hSPRY2) homologue in prostate cancer. Oncogene 24: 2166-2174, 2005.

18. Fritzsche S, Kenzelmann M, Hoffmann MJ, Müller M, Engers R, Gröne HJ and Schulz WA: Concomitant down-regulation of SPRY1 and SPRY2 in prostate carcinoma. Endocr Relat Cancer 13: 839-849, 2006

19. Lo TL, Yusoff P, Fong CW, Guo K, McCaw BJ, Phillips WA, Yang H Wong ES, Leong HF, Zeng Q, et al: The ras/mitogen-activated protein kinase pathway inhibitor and likely tumor suppressor proteins, sprouty 1 and sprouty 2 are deregulated in breast cancer. Cancer Res 64: 6127-6136, 2004.

20. Fong CW, Chua MS, McKie AB, Ling SH, Mason V, Li R, Yusoff P, Lo TL, Leung HY, So SK and Guy GR: Sprouty 2, an inhibitor of mitogen-activated protein kinase signaling, is down-regulated in hepatocellular carcinoma. Cancer Res 66: 2048-2058, 2006.
21. Masoumi-Moghaddam S, Amini A, Wei AQ, Robertson G and Morris DL: Sprouty 2 protein, but not Sprouty 4, is an independent prognostic biomarker for human epithelial ovarian cancer. Int J Cancer 137: 560-570, 2015.

22. Kwabi-Addo B, Wang J, Erdem H, Vaid A, Castro P, Ayala G and Ittmann M: The expression of Sprouty1, an inhibitor of fibroblast growth factor signal transduction, is decreased in human prostate cancer. Cancer Res 64: 4728-4735, 2004

23. Masoumi-Moghaddam S, Amini A, Wei AQ, Robertson G and Morris DL: Sprouty 1 predicts prognosis in human epithelial ovarian cancer. Am J Cancer Res 5: 1531-1541, 2015.

24. Rathmanner N, Haigl B, Vanas V, Doriguzzi A, Gsur A and Sutterlüty-Fall H: Sprouty2 but not Sprouty4 is a potent inhibitor of cell proliferation and migration of osteosarcoma cells. FEBS Lett 587: 2597-2605, 2013.

25. Jäggi F, Cabrita MA, Perl AK and Christofori G: Modulation of endocrine pancreas development but not beta-cell carcinogenesis by Sprouty4. Mol Cancer Res 6: 468-482, 2008.

26. Tennis MA, Van Scoyk MM, Freeman SV, Vandervest KM, Nemenoff RA and Winn RA: Sprouty-4 inhibits transformed cell growth, migration and invasion, and epithelial-mesenchymal transition, and is regulated by Wnt7A through PPARgamma in non-small cell lung cancer. Mol Cancer Res 8: 833-843, 2010.

27. Vanas V, Mühlbacher E, Kral R and Sutterlüty-Fall H: Sprouty 4 interferes with cell proliferation and migration of breast cancer-derived cell lines. Tumour Biol 35: 4447-4456, 2014.

28. Faratian D, Sims AH, Mullen P, Kay C, Um I, Langdon SP and Harrison DJ: Sprouty 2 is an independent prognostic factor in breast cancer and may be useful in stratifying patients for trastuzumab therapy. PLoS One 6: e23772, 2011.

29. Ding W, Bellusci S, Shi W and Warburton D: Genomic structure and promoter characterization of the human Sprouty4 gene, a novel regulator of lung morphogenesis. Am J Physiol Lung Cell Mol Physiol 287: 52-59, 2004.

30. Khaitan D, Dinger ME, Mazar J, Crawford J, Smith MA, Mattick JS and Perera RJ: The melanoma-upregulated long noncoding RNA SPRY4-IT1 modulates apoptosis and invasion. Cancer Res 71: 3852-3862, 2011.

31. Berger W, Elbling L, Hauptmann E and Micksche M: Expression of the multidrug resistance-associated protein (MRP) and chemoresistance of human non-small-cell lung cancer cells. Int J Cancer 73: 84-93, 1997.

32. Haigl B, Mayer CE, Siegwart G and Sutterlüty H: Sprouty4 levels are increased under hypoxic conditions by enhanced mRNA stability and transcription. Biol Chem 391: 813-821, 2010.

33. Kral RM, Mayer CE, Vanas V, Gsur A and Sutterlüty-Fall H: In non-small cell lung cancer mitogenic signaling leaves Sprouty1 protein levels unaffected. Cell Biochem Funct 32: 96-100, 2014.

34. Sirivatanauksorn Y, Sirivatanauksorn V, Srisawat C, Khongmanee A and Tongkham C: Differential expression of sprouty genes in hepatocellular carcinoma. J Surg Oncol 105: 273-276, 2012.

35. Wang J, Thompson B, Ren C, Ittmann M and Kwabi-Addo B: Sprouty4, a suppressor of tumor cell motility, is down regulated by DNA methylation in human prostate cancer. Prostate 66: 613-624, 2006

36. Doriguzzi A, Haigl B, Gsur A and Sutterlüty-Fall H: The increased Sprouty4 expression in response to serum is transcriptionally controlled by Specific protein 1 . Int J Biochem Cell Biol 64: 220-228, 2015.

37. Mayer CE, Haigl B, Jantscher F, Siegwart G, Grusch M, Berger W and Sutterlüty H: Bimodal expression of Sprouty2 during the cell cycle is mediated by phase-specific Ras/MAPK and c-Cbl activities. Cell Mol Life Sci 67: 3299-3311, 2010.

38. So WK, Cheng JC, Liu Y, Xu C, Zhao J, Chang VT and Leung PC: Sprouty4 mediates amphiregulin-induced down-regulation of E-cadherin and cell invasion in human ovarian cancer cells. Tumour Biol, 2016.

39. Zhang H, Guo Q, Wang X, Wang C, Zhao X and Li M: Aberrant expression of hSef and Sprouty4 in endometrial adenocarcinoma. Oncol Lett 11: 45-50, 2016. 